PROCEEDINGS OF THE

AMERICAN MATHEMATICAL SOCIETY

Volume 130, Number 5, Pages 1503-1506

S 0002-9939(01)06415-2

Article electronically published on December 20, 2001

\title{
ON AN ADJOINT FUNCTOR TO THE THOM FUNCTOR
}

\author{
YULI B. RUDYAK
}

(Communicated by Ralph Cohen)

\begin{abstract}
We construct a right adjoint functor to the Thom functor, i.e., to
\end{abstract} the functor which assigns the Thom space $T \xi$ to a vector bundle $\xi$.

\section{INTRODUCTION}

Let $\tau$ denote the functor which assigns the Thom space $T \xi$ to a vector bundle $\xi$, and similarly for maps. The goal of this paper is to construct the right adjoint functor $\lambda$ to the functor $\tau$.

To motivate this result, I remark that it is always nice to know whether a functor admits an adjoint one. However, here we have a more interesting motivation. Namely, it is useful to know when a space is the Thom space of a certain vector bundle (spherical fibration). For example, de-Thomification plays an important role in the theory of immersion of manifolds; see $[\mathrm{BP},[\mathrm{C}]$ and the survey $[\mathrm{L}]$. In fact, Brown and Peterson $[\mathrm{BP}$ de-Thomify a space, while Cohen $\mathrm{C}$ de-Thomifies a map. However, these de-Thomifications are very ad hoc. So, it is reasonable to want a de-Thomification machine, like the de-looping machine of May $[\mathrm{M}]$ or Boardman and Vogt [BV]. The following observation of Beck B plays the crucial role in the de-looping theory. The suspension functor $S$ is the left adjoint to the loop functor $\Omega$, and so there is a monad $M:=\Omega S$. Clearly, every loop space is a space over $M$. Conversely, if a space $X$ is a space over $M$, then, using the simplicial resolution of the $M$-space $X$, one can provide a de-looping of $X$ "at the simplicial level", and then certain additional arguments enable us to lift this "simplicial de-looping" to the geometric level; see $[\mathrm{B}], \mathrm{M}]$.

Here we have a dual situation. As usual, the functor $C:=\tau \lambda$ is a comonad, and every Thom space is a space over $C$. Conversely, if $X$ is a space over $C$ then, dually to what we said above, one can take the cosimplicial resolution of $X$ and provide a de-Thomification of $X$ "at the cosimplicial level". However, in order to do the next step, a lifting to the geometrical level, one must prove that the Thomification commutes with the functor Tot, and this problem looks quite complicated; cf. Bo.

Summarizing, one can consider this paper as a first step in an attack on the de-Thomification problem.

Notice that the above arguments enable us to prove that a certain space is not a Thom space: it suffices to check that it is not a space over the comonad $C$. For

Received by the editors April 2, 1999 and, in revised form, March 26, 2000.

2000 Mathematics Subject Classification. Primary 55R25; Secondary 18A40.

(C)2001 American Mathematical Society 
example, we have (implicitly) used these arguments in [R1] in order to prove that the spectra $k$ and $k O$ are not Thom spectra.

\section{THE CASE OF NON-ORIENTABLE BUNDLES}

Let $O_{n}$ be the group of orthogonal transformations of the Euclidean space $\mathbb{R}^{n}$, let $B O_{n}$ denote its classifying space, and let $\gamma$ denote the universal $n$-dimensional vector bundle over $B O_{n}$. Given a locally trivial bundle $\xi$ with the fiber $\mathbb{R}^{n}$ and structure group $O_{n}$, let $T \xi$ denote the Thom space of $\xi$, i.e., $T \xi:=D(\xi) / S(\xi)$, where $D(\xi)$ is the total space of the unit disc bundle and $S(\xi)$ is the total space of

the unit sphere subbundle of $D(\xi)$. We regard $T \xi$ as a pointed space with the base point given by $S(\xi)$.

Let $\mathcal{K}$ be the category whose objects are maps $f: B \rightarrow B O_{n}$, where $B$ is a connected space and $f$ is a map such that

$$
\pi_{1}(B) \stackrel{f_{*}}{\longrightarrow} \pi_{1}\left(B O_{n}\right)=\mathbb{Z} / 2
$$

is an epimorphism, and whose morphisms are commutative diagrams

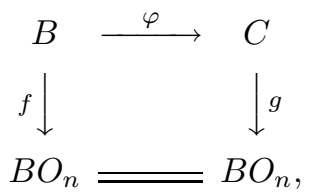

where $f$ and $g$ are objects of $\mathcal{K}$. Let $\mathcal{S}$ be the category whose objects are pointed spaces $X$ with $\pi_{i}(X)=0$ for $i<n$ and $\pi_{n}(X)=\mathbb{Z} / 2$, and whose morphisms are maps $f: X \rightarrow Y$ such that $f_{*}: \pi_{n}(X) \rightarrow \pi_{n}(Y)$ is an isomorphism.

Let $\tau: \mathcal{K} \rightarrow \mathcal{S}$ be the Thom functor which assigns the object $\tau f:=T\left(f^{*} \gamma\right) \in \mathcal{S}$ to the object $f: X \rightarrow B O_{n}$ of $\mathcal{K}$.

Theorem 1. The functor $\tau$ admits a right adjoint functor $\lambda: \mathcal{S} \rightarrow \mathcal{K}$.

Proof. We construct $\lambda$ as follows. Choose any $X \in \mathcal{S}$. Given an integer $k$, let $\Omega_{k}^{n} X$ be the component of $\Omega^{n} X$ corresponding to $k \in \pi_{n}(X)=\pi_{0}\left(\Omega^{n} X\right)=\mathbb{Z} / 2$. The standard $O_{n}$-action on $\mathbb{R}^{n}$ yields the obvious $O_{n}$-action on $S^{n}$, which, in turn, induces a (right) $O_{n}$-action on $\Omega^{n} X=(X, *)^{\left(S^{n}, *\right)}$, and it is clear that every component $\Omega_{k}^{n} X, k=0,1$, is $O_{n}$-invariant. Convert the right $O_{n}$-action on $\Omega_{1}^{n} X$ into a left $O_{n}$-action by setting $g a=a g^{-1}, g \in O_{n}, a \in \Omega_{1}^{n} X$. Consider the locally trivial bundle

$$
p: E O_{n} \times_{O_{n}} \Omega_{1}^{n} X \rightarrow B O_{n}
$$

which is associated with the universal principal $O_{n}$-bundle $\Gamma:=\left\{E O_{n} \rightarrow B O_{n}\right\}$; cf. [PS]. We define $\lambda X$ to be the map $p$. The $\lambda$-action on morphisms is clear.

We prove that $\lambda$ is right adjoint to $\tau$, i.e., that $\mathcal{K}(f, \lambda X)=\mathcal{S}\left(T\left(f^{*} \gamma\right), X\right)$ for every $f: B \rightarrow B O_{n}$; cf. [R2]. Indeed, consider the principal $O_{n}$-bundle

$$
f^{*} \Gamma=\{q: E \rightarrow B\},
$$

and let $\xi$ be the $\Omega_{1}^{n} X$-bundle associated with $f^{*} \Gamma$, i.e.

$$
\xi=\left\{E \times_{O_{n}} \Omega_{1}^{n} X \rightarrow B\right\} .
$$

Then $\xi$ is induced by $f$ from the bundle $\lambda X=\left\{p: E O_{n} \times_{O_{n}} \Omega_{1}^{n} X \rightarrow B O_{n}\right\}$. So, $\mathcal{K}(f, \lambda X)=\operatorname{Sec} \xi$, where $\operatorname{Sec} \xi$ denotes the set of all sections of $\xi$. 
For every $b \in B$ choose any $O_{n}$-equivariant map $i_{b}: O_{n} \rightarrow E$ with $q i_{b}\left(O_{n}\right)=b$. We have (the first equality can be found e.g. in $[\mathrm{H}]$ )

$$
\begin{aligned}
\operatorname{Sec} \xi= & \left\{O_{n} \text {-equivariant maps } E \rightarrow \Omega_{1}^{n} X\right\} \\
= & \left\{O_{n} \text {-equivariant maps } f: E \rightarrow(X, *)^{\left(S^{n}, *\right)}\right. \\
& \text { such that } \left.f(x) \in \Omega_{1}^{n} X \text { for every } a \in E\right\} \\
= & \left\{\text { maps } f: E \times_{O_{n}}\left(S^{n}, *\right) \rightarrow(X, *)\right. \text { such that the map } \\
& \quad\left(S^{n}, *\right)=O_{n} \times_{O_{n}}\left(S^{n}, *\right) \stackrel{i_{b}}{\longrightarrow} E \times_{O_{n}}\left(S^{n}, *\right) \stackrel{f}{\longrightarrow}(X, *) \\
& \text { belongs to } \left.\Omega_{1}^{n} X \text { for every } b\right\} \\
= & \mathcal{S}\left(T\left(f^{*} \gamma\right), X\right) .
\end{aligned}
$$

\section{THE CASE OF ORIENTABLE BUNDLES}

Let $B S O_{n}$ be the classifying space for the connected component $S O_{n}$ of $O_{n}$. Let $\mathcal{K}^{\prime}$ be the category whose objects are maps $f: B \rightarrow B S O_{n}$, where $B$ is a connected space, and whose morphisms are commutative diagrams

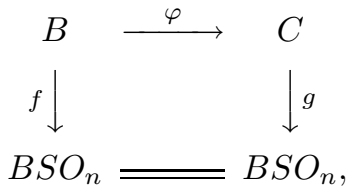

where $f$ and $g$ are objects of $\mathcal{K}^{\prime}$. Let $\mathcal{S}^{\prime}$ be the category whose objects are pairs $\left(X, a_{X}\right)$, where $X$ is a pointed space with $\pi_{i}(X)=0$ for $i<n$ and $a_{X}$ is a generator (one of two) of $\pi_{n}(X)=\mathbb{Z}$, and whose morphisms are maps $\varphi: X \rightarrow Y$ with $\varphi_{*}\left(a_{X}\right)=a_{Y}$.

Let $\gamma^{\prime}$ be the universal oriented $n$-dimensional vector bundle over $B S O_{n}$. There is a unique element $a \in \pi_{n}\left(T \gamma^{\prime}\right)=\mathbb{Z}$ such that $\langle u, h(a)\rangle=1$, where $u \in H^{n}\left(T \gamma^{\prime}\right)=$ $\mathbb{Z}$ is the orientation of $\gamma^{\prime}, h: \pi_{n}\left(T \gamma^{\prime}\right) \rightarrow H_{n}\left(T \gamma^{\prime}\right)$ is the Hurewicz homomorphism and $\langle-,-\rangle$ is the Kronecker pairing.

Given an object $f: X \rightarrow B S O_{n}$ of $\mathcal{K}^{\prime}$, we have the canonical map $F: T\left(f^{*} \gamma^{\prime}\right) \rightarrow$ $T \gamma^{\prime}$, and $F_{*}: \mathbb{Z}=\pi_{n}\left(T\left(f^{*}(\gamma)\right)\right) \rightarrow \pi_{n}(T \gamma)=\mathbb{Z}$ is an isomorphism. Now define the Thom functor $\tau^{\prime}: \mathcal{K}^{\prime} \rightarrow \mathcal{S}^{\prime}$ by setting $\tau^{\prime} f=\left(T\left(f^{*} \gamma\right),\left(F_{*}\right)^{-1}(a)\right)$.

Theorem 2. The functor $\tau^{\prime}$ admits a right adjoint functor $\lambda^{\prime}: \mathcal{S}^{\prime} \rightarrow \mathcal{K}^{\prime}$.

Proof. Given an object $\left(X, a_{X}\right)$ of $\mathcal{K}^{\prime}$, consider the isomorphism $\pi_{n}(X) \cong \pi_{0}\left(\Omega^{n} X\right)$, and let $\Omega_{1}^{n} X$ be the component of $\Omega^{n} X$ which corresponds to $a_{X}$. As in $\S 1$, we have the left $S O_{n}$-action on $\Omega^{n} X$, and clearly the component $\Omega_{1}^{n} X$ is invariant under the $S O_{n}$-action on $\Omega^{n} X$. We construct a fibre bundle $p: E S O_{n} \times_{S O_{n}} \Omega_{1}^{n} X \rightarrow B S O_{n}$, and we define $\lambda^{\prime}\left(X, a_{X}\right):=p$. Now the proof can be completed similarly to that of Theorem 1.

\section{REFERENCES}

[B] J. Beck, On H-spaces and infinite loop spaces, Category Theory, Homotopy Theory and Their Applications III. Lecture Notes in Mathematics 99, Springer, Berlin Heidelberg New York, 1969, pp. 139-153. MR 40:2079 
[BV] J.M. Boardman, R.M.Vogt, Homotopy invariant algebraic structures on topological spaces, Lecture Notes in Mathematics 347, Springer, Berlin Heidelberg New York, 1973. MR 54:8623a

[Bo] A.K. Bousfield, On the homology spectral sequence of a cosimplicial space, Amer. J.Math. 109 (1987), 361-394. MR 88j:55017

[BP] E.H. Brown, F.P. Peterson, A universal space for normal bundles of $n$-manifolds, Comment. Math. Helv. 54 (1979), 405-430. MR 80k:57055

[C] R. Cohen, The immersion conjecture for differentiable manifolds., Ann of Math 122 (1985), 237-328. MR 86m:57030

$[H]$ D. Husemoller, Fibre bundles. - McGraw-Hill, New York, 1966. MR 37:4821

[L] J. Lannes, La conjecture des immersions (d'après R.L. Cohen, E.H.Brown, F.P.Peterson et al.), Bourbaki Seminar 1981/1982, Soc. Math. France, Paris, 1982, pp. 331-346. MR 84i:57020

[M] J.P. May, The geometry of iterated loop spaces, Lecture Notes in Mathematics 271, Springer, Berlin Heidelberg New York, 1972. MR 54:8623b

[PS] R. Patterson, R. Stong, Orientability of bundles, Duke Math. J. 39 (197), 619-622. MR 47:4248

[R1] Yu. Rudyak, The spectra $k$ and $k O$ are not Thom spectra, Group Representations: Cohomology, Group Actions and Topology, Proc. Symp. Pure Math. 63, Amer. Math. Soc, Providence, RI, 1998, pp. 475-483. MR 98m:55007

[R2] Yu. Rudyak, On Thom Spectra, Orientability and Cobordism, Springer, Berlin, 1998. MR 99f:55001

Mathematisches Institut, Universität Heidelberg, Im Neuenheimer Feld 288, 69120 HeIDELBERG, GeRmany

E-mail address: rudyak@mathi.uni-heidelberg.de

Current address: Department of Mathematics, University of Florida, Gainesville, Florida 32611-8105

E-mail address: rudyak@math.ufl.edu 